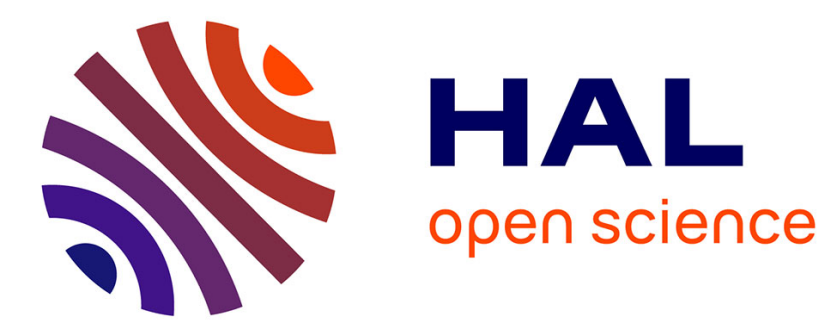

\title{
Alpha rebound improves on-line detection of the end of motor imageries
}

\author{
Cecilia Lindig-León, Laurent Bougrain, Sébastien Rimbert
}

\section{To cite this version:}

Cecilia Lindig-León, Laurent Bougrain, Sébastien Rimbert. Alpha rebound improves on-line detection of the end of motor imageries. IEEE EMBS Neural engineering conference, Apr 2015, Montpellier, France. hal-01092284v2

\section{HAL Id: hal-01092284 \\ https://inria.hal.science/hal-01092284v2}

Submitted on 20 Feb 2015

HAL is a multi-disciplinary open access archive for the deposit and dissemination of scientific research documents, whether they are published or not. The documents may come from teaching and research institutions in France or abroad, or from public or private research centers.
L'archive ouverte pluridisciplinaire HAL, est destinée au dépôt et à la diffusion de documents scientifiques de niveau recherche, publiés ou non, émanant des établissements d'enseignement et de recherche français ou étrangers, des laboratoires publics ou privés. 


\title{
Alpha rebound improves on-line detection of the end of motor imageries
}

\author{
Cecilia Lindig-León ${ }^{1,2}$, Laurent Bougrain ${ }^{2,1}$ and Sébastien Rimbert ${ }^{2}$
}

\begin{abstract}
Limb movement execution or imagination induce sensorimotor rhythms that can be detected in electroencephalographic (EEG) recordings. This article presents the interest of considering not only the beta frequency band but also the alpha band to detect the elicited EEG rebound, i.e. the increasing of oscillatory power synchronization, at the end of motor imageries. From database $2 \mathrm{a}$ of the BCI competition IV, it is shown that this phenomenon can be stronger over the alpha than the beta band and it is experimentally demonstrated that the analysis on the alpha band improves the detection of the end of motor imageries. Moreover a variant method to compute the oscillatory power without referring to a baseline period is proposed; such capacity is useful for self-paced brain-computer interfaces (BCI) control.
\end{abstract}

\section{INTRODUCTION}

Brain activity recorded over the primary sensorimotor cortex presents an oscillatory behavior that is of particular interest within two specific frequency bands: the alpha (8$13 \mathrm{~Hz})$ and beta (13-25 Hz) bands [1], [2], [3], [4], as they have been shown to be modulated during and following the preparation and execution of voluntary movement, passive movement, imagined movement, and even tactile stimulation [5]. This modulation takes two different forms: a reduction in oscillatory power within alpha and beta bands known as event-related desynchronization (ERD) originated over contralateral sensorimotor areas and beginning approximately 2 seconds prior to movement initiation; and an event-related synchronization (ERS) that follows the movement termination and that persists for hundred milliseconds [6]. Although this later modulation is known to be specific of the beta band and is denoted as post-movement beta rebound [7], [8]; in the present study it is observed that this phenomenon is enhanced when analyzed in the alpha range, since the modulation amplitude is larger than the one observed in the beta band and its morphology shows increased variations between the post-movement condition and the remainder states, leading to a more reliable detection of the end of imagined movements that becomes relevant for selecting suitable commands to control BCIs.

The presence of this modulation within the alpha band was already detected as a response to action observation and execution of motor neurons [5], it was reported as an increasing amplitude in terms of the relative power (ERS\%) with respect to a baseline period registered some seconds before the event. Nevertheless, the requirement of a resting

\footnotetext{
${ }^{1}$ Inria, $\quad$ Villers-lès-Nancy, F-54600, France cecilia.lindig-leoneinria.fr

2 Université de Lorraine, LORIA, UMR 7503, Vandœuvre-lèsNancy, F-54506, Francelaurent.bougraineloria.fr, sebastien.rimbert1detu.univ-lorraine.fr
}

state period prior to movement imagination to obtain a reference interval, represents an important drawback in the BCI context. Especially for a self-paced control, in which the user triggers the command independently of the system and there is no warning of the incoming action, so that each state has to be straightaway detected. A way to tackle this difficulty consists of registering a baseline period at the start of each session to use it as reference interval to analyze subsequent signal segments. However, this approach is not so accurate for long recordings due to changes in the user mental state caused by fatigue; along with any kind of visual or auditory stimuli occurring in the environment and variations in the electrodes impedance and position. To address this inconvenience, in the present study a variant method to compute the oscillatory power without referring to a baseline period is proposed, so that the EEG segments can be analyzed independently favoring the selection of commands for self-paced BCIs.

\section{MATERIAL}

To illustrate the proposed method EEG recordings from 9 healthy subjects taken from database $2 \mathrm{a}$ of the BCI competition IV [9] were used. Each subject participated in 2 different sessions performing four different motor imageries: left hand, right hand, feet and tongue. For the purposes of this study which are not focused on motor imagery classification but on detection of motor imagery stopping, only 2 classes (left hand and right hand) were considered. Each session contains 6 runs comprising 12 trials per class, which yields a total of 144 trials per task. Signals were recorded with a sampling rate of $250 \mathrm{~Hz}$ over 22 electrodes and examined by an expert in order to label trials containing artifacts (see [10], [11] for details).

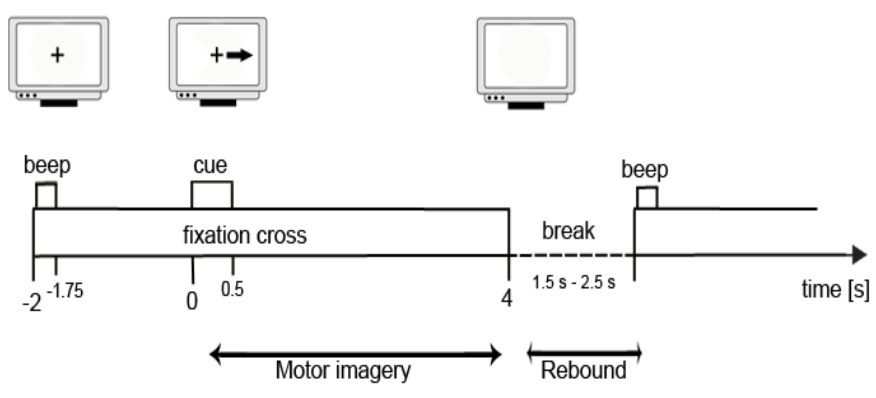

Fig. 1. Timing Scheme of the paradigm. The motor imagery is performed from $0 \mathrm{~s}$ (cue) to $4 \mathrm{~s}$ (end of the fixation cross). The duration of the break between trials is variable from 1.5 to $2.5 \mathrm{~s}$.

Fig. 1 shows the timing scheme of the paradigm for each 
trial, which has an approximate duration of 8 seconds. During the first 2 seconds previous to the cue indicating the motor imagery to be performed the subject is in a resting state, which allows to observe a period of approximately 4 seconds after the interruption of the motor imagery before the next trial is starting.

\section{METHODS}

The purpose of this study is twofold. First, it prompts that at the end of motor imageries an increased synchronization in the alpha frequency band is elicited. The characteristics of this rebound (latency and amplitude) are drawn out and described from the EEG signals comprised in the used database. Second, it shows that it is advantageous to take into account this frequency range to improve the online detection of the end of an imagined movement. In this section two processing stages are presented, the first one describes the proposed method to compute the oscillatory power that allows observing the alpha rebound along the EEG signals; and the second one illustrates the classification method based on a single trial detection to identify the end of motor imageries.

\section{A. Quantifying the alpha rebound}

To get started, the trials that were found to contain artifacts were removed so that the neurophysiological phenomenon can be studied as clear as possible without considering unrelated variations to the oscillatory power synchronization in the alpha and beta bands (i.e. head muscles activity, electrodes displacement, etc.). The remaining trials were extracted starting 2 seconds before the cue and stopping 8 seconds after; thus, each one of them corresponds to a 10s-window over 22 channels (see Fig. 3a). This window size allows to observe the activity up to 4 seconds after the motor imagery was interrupted. The last trial of each run has been also removed due to the fact that the window size used to compute the activity overpasses the length of the run. The next step consists of the filtering of each trial using a 5th-order band-pass Butterworth filter. From this point on, the described analysis is carried out separately for the alpha and beta bands, which were considered in the ranges [8-13 Hz] and [13-25 Hz] respectively; a wider range including both alpha and beta bands is also presented as a plausible alternative to detect the end of motor imageries (see Fig. 3b). To measure the synchronization after the interruption of the imagined movement, the power of each trial was computed by squaring the amplitude of the samples (see Fig. 3c) and then by averaging the power samples across all trials (see Fig. 3d). Finally, the resulting signal was smoothed to reduce variability using a 1-second-sliding window with a $100 \mathrm{~ms}$ shifting step to compute the mean across samples (see Fig. 3e). This last step differs from [6]. Usually, the mean of the trials' power is normalized by the mean of the power of a baseline window as it is stated in the classic formula [mean power $\left._{\text {StudiedWindow }}\right)-$ mean (power Baseline]/mean $\left(\right.$ power $\left._{\text {Baseline }}\right)$ that leads to ERD/ERS percentages showing the evolution of the signal dynamics [6].

\section{B. On-line single trial detection of the alpha rebound}

In this stage all trials, even those labeled as containing artifacts, were kept in order to recreate the online recording conditions to propose a suitable method based on a single trial detection. At this point it can be observed that both, alpha and beta rebounds, are elicited just at the interruption of the imagined movement (second 4, see Fig. 3e) and that it has a latency of 3 seconds approximately. Thus, for the online detection, it has been decided to analyze trials using a 3-seconds sliding window with a 1-second-shifting step to create segments belonging to one of two different classes according to the occurring condition (i.e., segments with rebound and segments without it). Under this scheme, each trial contributes with 7 segments $([-1.5,1.5 \mathrm{~s}] ;[-0.5,2.5$ $\mathrm{s}] ;[0.5,3.5 \mathrm{~s}] ;[1.5,4.5 \mathrm{~s}] ;[2.5,5.5 \mathrm{~s}] ;[3.5,6.5 \mathrm{~s}])$, from which the first five are considered as non-target segments and the latest two as target segments. From session 1 a training dataset was generated to build a linear discriminant analysis (LDA) classifier, and from session 2 an unseen-data validation dataset was gathered up to test the system; this procedure was carried out separately for left hand and right hands.

\section{RESULTS}

With the aim of detecting the end of motor imageries, the present study described a method to compute the oscillatory power along the EEG signals and reveal the post-movement rebound associated with the event-related synchronization. This computation is not referred to a baseline period and variations in the signal power can be straightway detected, which is ideal for self-paced BCI systems. Moreover, it was observed that the amplitude and morphology of the postmovement rebound are boosted within the alpha band and that variations between this condition and the remainder brain states are bigger than in the beta band, leading to a more reliable classification (see Fig. 2 and Fig. 3e).
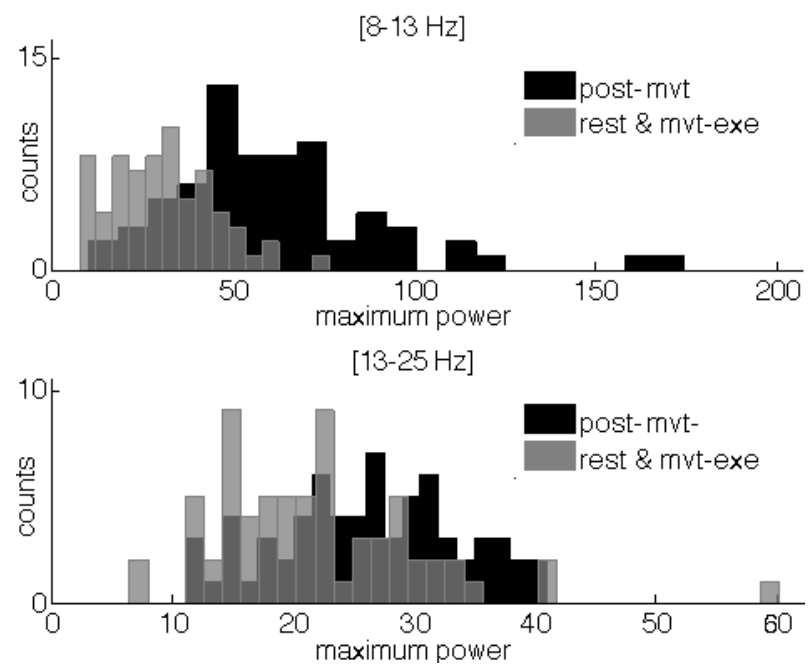

Fig. 2. Histograms of the highest oscillatory power values observed during post-movement periods (dark grey) and the remainder brain states (light grey) in the alpha band (upper) and the beta band (lower). Overlapping is shown in medium grey. 
TABLE I

CLASSIFICATION PERFORMANCE FOR ALL SUBJECTS USING THE UNSEEN SESSION 2 FOR LEFT HAND.

\begin{tabular}{|c|c|c|c|c|c|c|c|c|c|c|c|c|}
\hline \multicolumn{9}{|c|}{ Alpha band } & \multicolumn{3}{c|}{ Beft hand } \\
\hline \multirow{2}{*}{ Subject } & \multicolumn{3}{|c|}{ band } & \multicolumn{3}{c|}{ Alpha-beta band } \\
\hline & Acuracy & Sensitivity & Specificity & Precision & Acuracy & Sensitivity & Specificity & Precision & Acuracy & Sensitivity & Specificity & Precision \\
2 & 74.46 & 0.76 & 0.62 & 0.93 & 73.81 & 0.78 & 0.56 & 0.88 & 78.36 & 0.79 & 0.75 & 0.95 \\
2 & 72.51 & 0.80 & 0.52 & 0.82 & 56.28 & 0.73 & 0.31 & 0.61 & 56.71 & 0.83 & 0.37 & 0.49 \\
3 & 89.39 & 0.91 & 0.85 & 0.95 & 81.39 & 0.83 & 0.76 & 0.94 & 89.61 & 0.91 & 0.87 & 0.96 \\
4 & 67.53 & 0.76 & 0.42 & 0.80 & 70.56 & 0.78 & 0.48 & 0.82 & 70.56 & 0.79 & 0.48 & 0.81 \\
5 & 71.65 & 0.75 & 0.51 & 0.91 & 61.69 & 0.77 & 0.38 & 0.65 & 67.75 & 0.77 & 0.43 & 0.79 \\
6 & 75.97 & 0.79 & 0.63 & 0.91 & 69.26 & 0.74 & 0.44 & 0.87 & 77.92 & 0.79 & 0.70 & 0.93 \\
7 & 87.23 & 0.92 & 0.77 & 0.90 & 85.50 & 0.98 & 0.67 & 0.82 & 89.61 & 0.96 & 0.77 & 0.89 \\
8 & 78.36 & 0.81 & 0.69 & 0.92 & 72.29 & 0.76 & 0.53 & 0.91 & 81.39 & 0.82 & 0.78 & 0.95 \\
9 & 81.60 & 0.83 & 0.76 & 0.94 & 73.16 & 0.81 & 0.53 & 0.82 & 80.74 & 0.82 & 0.75 & 0.94 \\
\hline AVG & 77.63 & 0.81 & 0.64 & 0.90 & 71.55 & 0.80 & 0.52 & 0.81 & 76.96 & 0.83 & 0.66 & 0.86 \\
\hline
\end{tabular}

TABLE II

CLASSIFICATION PERFORMANCE FOR ALL SUBJECTS USING THE UNSEEN SESSION 2 FOR RIGHT HAND.

\begin{tabular}{|c|c|c|c|c|c|c|c|c|c|c|c|c|}
\hline \multicolumn{10}{|c|}{ Right hand } \\
\hline \multirow{2}{*}{ Subject } & Acuracy & Sensitivity & Specificity & Precision & Acuracy & Sensitivity & Specificity & Precision & Acuracy & Sensitivity & Specificity & Precision \\
\hline 1 & 80.12 & 0.80 & 0.79 & 0.96 & 74.33 & 0.78 & 0.58 & 0.89 & 80.54 & 0.81 & 0.76 & 0.94 \\
2 & 73.91 & 0.80 & 0.55 & 0.84 & 57.56 & 0.74 & 0.32 & 0.63 & 65.84 & 0.82 & 0.43 & 0.67 \\
3 & 87.58 & 0.86 & 0.93 & 0.98 & 80.95 & 0.80 & 0.85 & 0.97 & 88.61 & 0.87 & 0.96 & 0.99 \\
4 & 72.26 & 0.75 & 0.53 & 0.91 & 71.01 & 0.79 & 0.49 & 0.81 & 77.43 & 0.82 & 0.63 & 0.88 \\
5 & 71.22 & 0.74 & 0.49 & 0.91 & 70.19 & 0.77 & 0.47 & 0.83 & 73.50 & 0.78 & 0.55 & 0.87 \\
6 & 80.33 & 0.80 & 0.82 & 0.97 & 74.12 & 0.78 & 0.58 & 0.90 & 80.12 & 0.81 & 0.76 & 0.94 \\
7 & 84.06 & 0.85 & 0.80 & 0.94 & 87.37 & 0.96 & 0.72 & 0.86 & 85.30 & 0.93 & 0.71 & 0.86 \\
8 & 83.02 & 0.84 & 0.80 & 0.94 & 71.01 & 0.74 & 0.48 & 0.92 & 80.75 & 0.82 & 0.74 & 0.93 \\
9 & 77.43 & 0.79 & 0.70 & 0.94 & 71.43 & 0.78 & 0.50 & 0.83 & 79.50 & 0.80 & 0.75 & 0.95 \\
\hline AVG & 78.88 & 0.80 & 0.71 & 0.93 & 73.11 & 0.79 & 0.55 & 0.85 & 79.07 & 0.83 & 0.70 & 0.89 \\
\hline
\end{tabular}

Table I shows the classification performance in terms of accuracy, sensitivity, specificity and precision for the postmovement detection during left hand motor imageries for three different frequency bands: alpha, beta and alpha/beta. Trials from session 1 were used as training dataset to create a LDA classifier that separates data from both conditions and, trials from session 2, were used as evaluation dataset to evaluate the system performance. The same procedure was carried out for post-movement detection during right hand motor imageries (see Table II).

\section{CONCLUSIONS}

The present study shows the convenience of analyzing the oscillatory power within the alpha band to detect the postmovement rebound related to the end of motor imageries. Using dataset $2 \mathrm{a}$ of the BCI competition IV, the accuracy for post-movement rebound detection is 5\% superior over the alpha band than the beta band for almost all subjects and precision values increased around 10\%. Classification performance when both bands are considered together is also higher than the obtained within the beta range, nevertheless using only the alpha band is still more promising. These results show that the proposed online method is convenient for detecting the end of motor imageries; furthermore, it proposes a scheme to compute the oscillatory power without referring to a baseline period, which is of particular interest for self-paced BCI control.

\section{REFERENCES}

[1] P. G., "Central beta rhythm during sensorimotor activities in man," Electroencephalogr Clin Neurophysiol, vol. 51, no. 3, pp. 253-64, March 1981.
[2] C. Toro, G. Deuschl, R. Thatcher, S. Sato, C. Kufta, and M. Hallett, "Event-related desynchronization and movement-related cortical potentials on the ecog and eeg," Electroencephalogr Clin Neurophysiol, vol. 93, no. 5, pp. 380-9, Oct 1994.

[3] G. Pfurtscheller, A. Stancák, Jr, and G. Edlinger, "On the existence of different types of central beta rhythms below $30 \mathrm{hz}$," Electroencephalogr Clin Neurophysiol, vol. 102, no. 4, pp. 316-25, Apr 1997.

[4] C. Babiloni, F. Babiloni, F. Carducci, F. Cincotti, G. Cocozza, C. Del Percio, D. V. Moretti, and P. M. Rossini, "Human cortical electroencephalography (eeg) rhythms during the observation of simple aimless movements: a high-resolution eeg study," Neuroimage, vol. 17, no. 2, pp. 559-72, Oct 2002.

[5] P. Avanzini, M. Fabbri-Destro, R. Dalla Volta, E. Daprati, G. Rizzolatti, and G. Cantalupo, "The dynamics of sensorimotor cortical oscillations during the observation of hand movements: an eeg study," PLoS One, vol. 7, no. 5, p. e37534, 2012.

[6] G. Pfurtscheller and F. H. Lopes da Silva, "Event-related eeg/meg synchronization and desynchronization: basic principles," Clin Neurophysiol, vol. 110, no. 11, pp. 1842-57, Nov 1999.

[7] G. Pfurtscheller, A. Stancák, Jr, and C. Neuper, "Post-movement beta synchronization. a correlate of an idling motor area?" Electroencephalogr Clin Neurophysiol, vol. 98, no. 4, pp. 281-93, Apr 1996.

[8] M. T. Jurkiewicz, W. C. Gaetz, A. C. Bostan, and D. Cheyne, "Postmovement beta rebound is generated in motor cortex: Evidence from neuromagnetic recordings," Neurolmage, vol. 32, no. 3, pp. 1281 $1289,2006$.

[9] M. Tangermann, K.-R. Müller, A. Aertsen, N. Birbaumer, C. Braun, C. Brunner, R. Leeb, C. Mehring, K. J. Miller, G. R. Müller-Putz, G. Nolte, G. Pfurtscheller, H. Preissl, G. Schalk, A. Schlögl, C. Vidaurre, S. Waldert, and B. Blankertz, "Review of the bci competition iv," Front Neurosci, vol. 6, p. 55, 2012.

[10] M. Naeem, C. Brunner, R. Leeb, B. Graimann, and G. Pfurtscheller, "Seperability of four-class motor imagery data using independent components analysis," J Neural Eng, vol. 3, no. 3, pp. 208-16, Sep 2006.

[11] C. Brunner, M. Billinger, C. Vidaurre, and C. Neuper, "A comparison of univariate, vector, bilinear autoregressive, and band power features for brain-computer interfaces," Med Biol Eng Comput, vol. 49, no. 11, pp. 1337-46, Nov 2011. 
Raw EEG signal, subject 8

A)

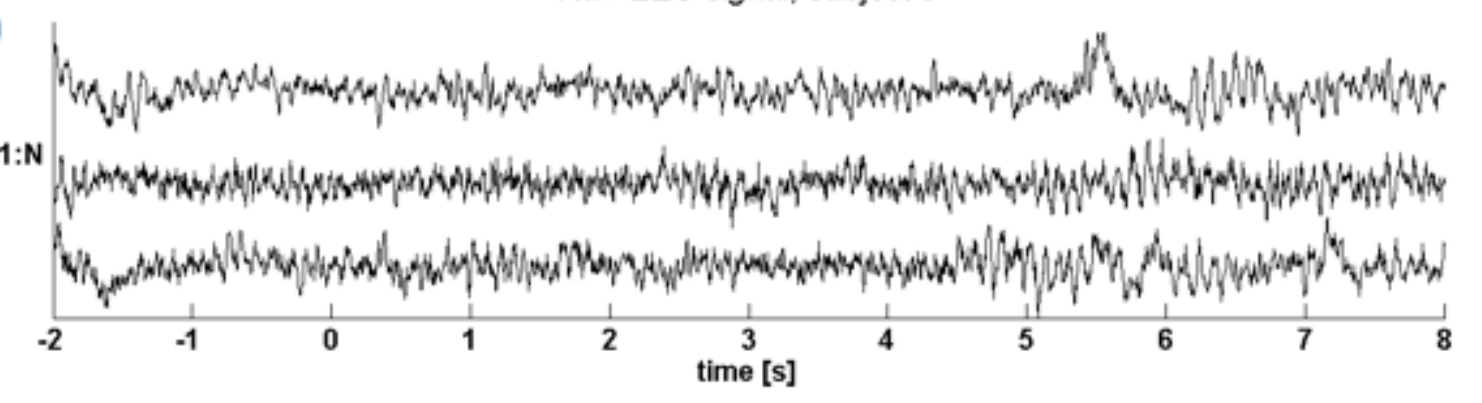

Bandpass-filtered EEG signals Bandpass-filtered EEG signals Bandpass-filtered EEG signals

B)

$(8-13 \mathrm{~Hz})$

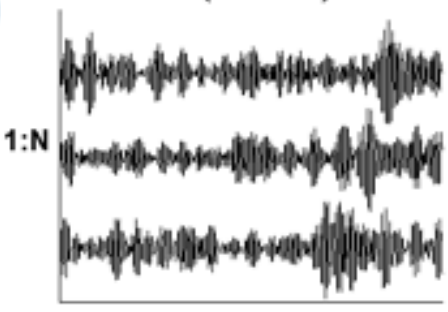

Squaring

C)

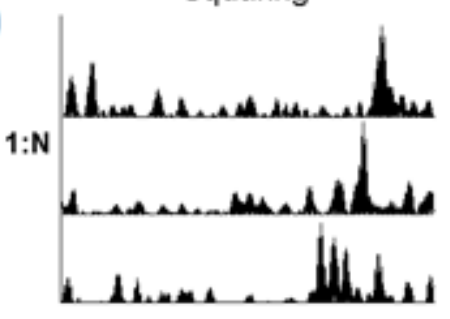

D) 120 Averaging over $N$ trials

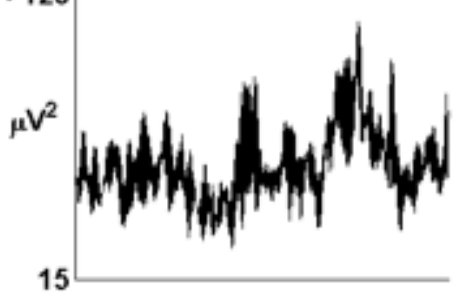

E)

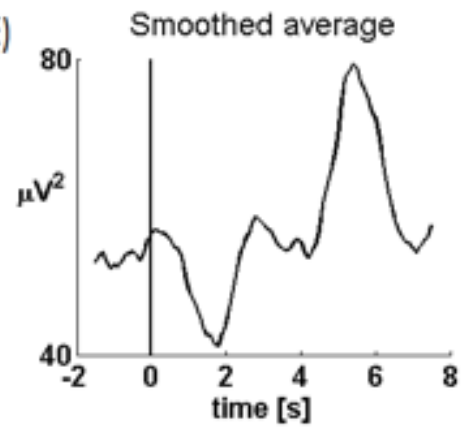

(13-25 Hz)

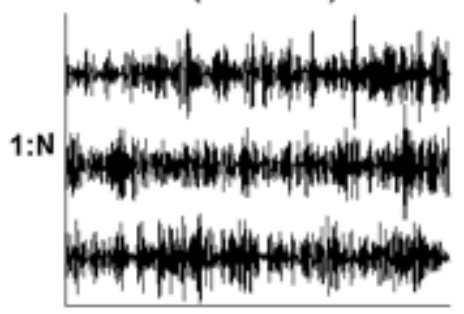

Squaring

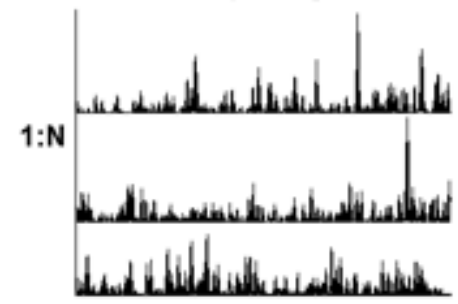

Averaging over $\mathrm{N}$ trials
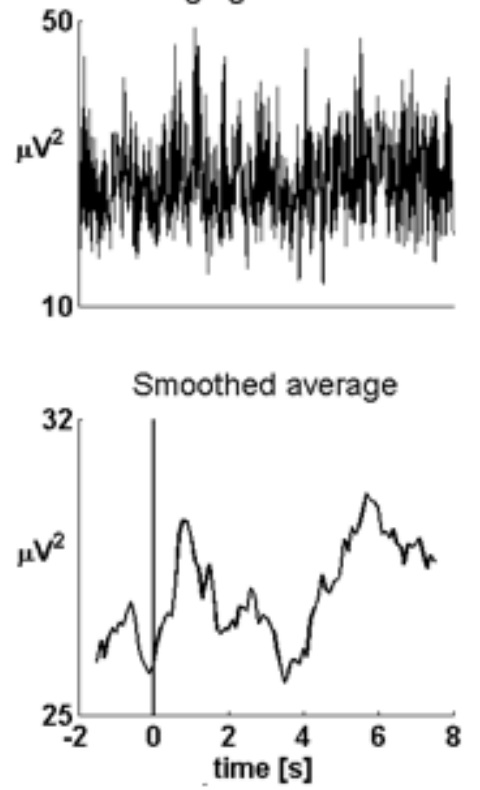

(8-25 Hz)

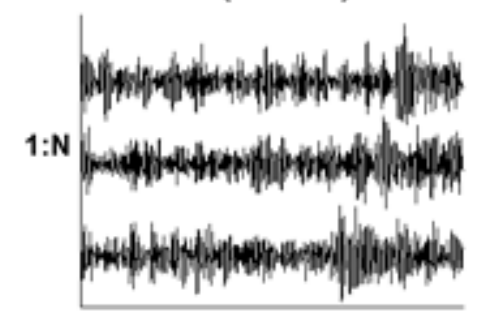

Squaring

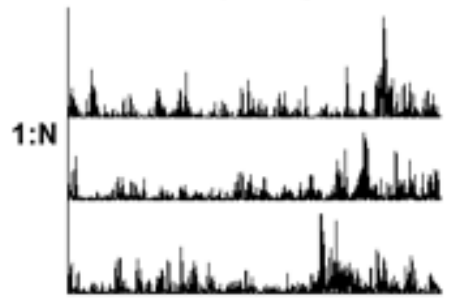

Averaging over $\mathrm{N}$ trials
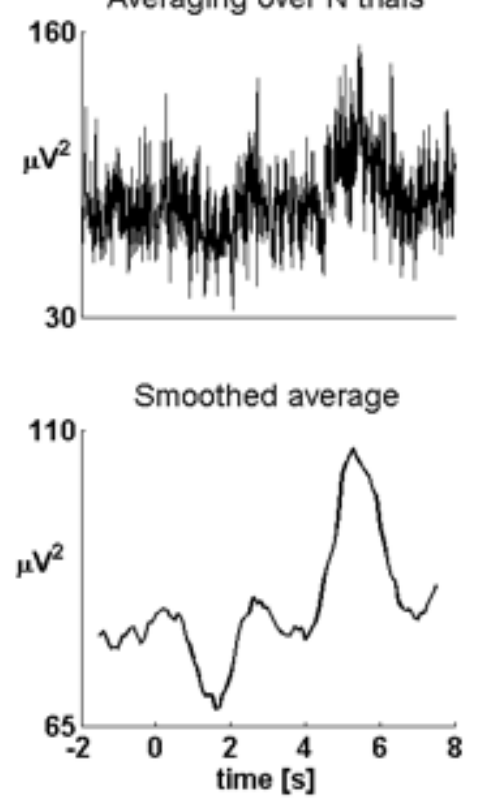

Fig. 3. Processing method to observe the oscillatory power synchronization of the (alpha, beta and alpha+beta) rebounds after the end of the motor imagery. The showed EEG segments correspond to left hand motor imageries recorded on electrode C4 for subject 8 